\title{
Spatially heterogeneous pressure raises risk of catastrophic shifts
}

\author{
Florian D. Schneider ${ }^{1,2} \cdot$ Sonia Kéfi ${ }^{1}$
}

Received: 11 May 2015 / Accepted: 30 November 2015 / Published online: 19 December 2015

(C) The Author(s) 2015. This article is published with open access at Springerlink.com

\begin{abstract}
Ecosystems may exhibit catastrophic shifts, i.e. abrupt and irreversible responses of ecosystem functions and services to continuous changes in external conditions. The search for early warning signs of approaching shifts has so far mainly been conducted on theoretical models assuming spatially-homogeneous external pressures (e.g. climatic). Here, we investigate how a spatially explicit pressure may affect ecosystems' risk of catastrophic shifts and the associated spatial early-warning signs. As a case study, we studied a dryland vegetation model assuming 'associational resistance', i.e. the mutual reduction of local grazing impact by neighboring plants sharing the investment in defensive traits. Consequently, grazing pressure depends on the local density of plants and is thus spatially-explicit. We focus on the distribution of vegetation patch sizes, which can be assessed using remote sensing and are candidate early warning signs of catastrophic shifts in drylands. We found that spatially explicit grazing affected both the resilience and the spatial patterns of the landscape. Grazing impact became self-enhancing in more fragmented landscapes, disrupted patch growth and put apparently 'healthy' drylands under high risks of catastrophic shifts. Our study highlights that a spatially explicit pressure may affect the
\end{abstract}

Florian D. Schneider

florian.schneider@univ-montp2.fr

Sonia Kéfi

sonia.kefi@univ-montp2.fr

1 Institut des Sciences de l'Evolution, Université de Montpellier, CNRS, IRD, EPHE, CC065,

Place Eugène Bataillon, 34095 Montpellier

Cedex 05, France

2 Senckenberg Biodiversity and Climate Research Centre (BiK-F), Senckenberganlage 25, 60325 Frankfurt am Main, Germany nature of the spatial pattern observed and thereby change the interpretation of the early warning signs. This may generalize to other ecosystems exhibiting self-organized spatial patterns, where a spatially-explicit pressure may interfere with pattern formation.

Keywords Catastrophic shifts $\cdot$ Spatial patterns . Grazing · Desertification · Early warning signs ·

Patch size distribution

\section{Introduction}

Many ecosystems respond in an abrupt manner to a gradually increasing pressure (Suding et al. 2004; Suding and Hobbs 2009), a phenomenon which has been referred to as 'catastrophic shifts' in the literature (Holling 1973; Scheffer et al. 2001). Identifying reliable indicators of imminent catastrophic shifts would help to prevent irreversible degradation and thus improve sustainable ecosystem management. Recent model analyses suggest that a series of so-called generic early warning signals in time (Scheffer et al. 2009; Dakos et al. 2012) and space (Scheffer et al. 2009; Kéfi et al. 2014) can be used to forecast decreasing ecosystem resilience, where 'resilience' refers to the magnitude of disturbance that an ecosystem can endure without experiencing a catastrophic shift (Gunderson 2000). In particular, metrics of spatial structure have been discussed as indicators of degradation in ecosystems exhibiting a clear spatial organisation, such as drylands (Rietkerk et al. 2004; Kéfi et al. 2007b, 2014; Bailey 2011). In these ecosystems, aggregation of individuals into patches (i.e. clustering) becomes more pronounced with increasing pressure, and the distribution of patch-sizes deviates increasingly from 
a pure power-law as the largest patches fragment into smaller ones (Kéfi et al. 2007b, 2014; Lin et al. 2010; Moreno-de las Heras et al. 2011). However, some experimental studies have not confirmed the expected trends in spatial patterns along gradients of pressure (Cline et al. 2014) or have been subject to important noise in the data (Carpenter et al. 2011). Therefore, to make use of spatial indicators, it is important to identify the conditions under which spatial pattern can be an informative indicator of ecosystem degradation.

The great majority of the model approaches that have contributed to the identification of early warning signals have assumed the existence of locally constrained, positive feedback mechanisms on recruitment or growth of individuals (Callaway 1995; van de Koppel et al. 1997; Aguiar and Sala 1999; Puigdefábregas 2005; Borgogno et al. 2009). These local feedbacks have been shown to play a crucial role in both the emergence of spatial patterns and ecosystem resilience (Guichard et al. 2003; Rietkerk et al. 2004; Barbier et al. 2006; Kéfi et al. 2007b).

At the same time, these models typically consider external pressures, e.g. mortality by consumption or disease (Rietkerk et al. 2002; Kéfi et al. 2007b, 2011; Manor and Shnerb 2008; von Hardenberg et al. 2010), to be homogeneous in space, meaning that they are affecting all individuals equally. However, many types of pressures are likely to include positive local feedback mechanisms that render the pressure spatially heterogeneous, i.e. cases where the intensity of the pressure depends on the local density of individuals. For instance, the physical damage caused by heavy storms on trees is highest next to openings in the forest canopy (Kubo et al. 1996; Pascual and Guichard 2005). This mechanism explains the robust formation of scale-free gap clusters in forests (Pascual and Guichard 2005). Similar scale-free patterns occur in wave-disturbed intertidal mussel beds where biotic interactions determine local chances of establishment and where the abiotic, physical impact of waves is highest on mussels at the edge of a gap, due to their reduced byssal thread attachment to the ground (Guichard et al. 2003). Also, predator-prey dynamics in a spatially explicit context lead to clustering of the prey because of the density-dependent reduction of individual feeding pressure (Pascual and Guichard 2005). Including spatially heterogeneous pressures in models of pattern formation should therefore be of great relevance for our understanding of ecosystem dynamics as well as the relevant indicators of ecological resilience.

This paper aims at addressing the effect of a spatially explicit pressure on ecosystem resilience, emergent spatial patterns and the possible use of these patterns as indicators of loss of ecosystem resilience. Therefore, we chose to investigate the effect of livestock grazing on the vegetation of arid ecosystems as a case study. Grazing, a major factor of desertification in large parts of the world (Asner et al. 2004; Millennium Ecosystem Assessment 2005), can have a strong spatial component (Callaway 1995; Bailey et al. 1996; WallisDeVries et al. 1999; van de Koppel et al. 2002). In arid shrublands that have historically been exposed to grazing by large herbivores, most plant species have developed mechanical defenses against large herbivores, such as fast regrowth from the root stock, indigestible tissue, the development of prostrate growth forms and the evolution of spines and thorns (Lucas et al. 2000;Días 2007).

Thus, coinciding with the effects of abiotic facilitation, i.e. the amelioration of the local environmental conditions through shading or water retention by the presence of vegetation (Milchunas and Noy-Meir 2002), the canopy of plants with defenses against large herbivores also provides refugia from large herbivores to neighboring plants (Milchunas and Noy-Meir 2002; Baraza et al. 2006; Graff et al. 2007; Barbosa et al. 2009). Similar to the examples of mussel beds and forest gaps named above, this mechanism leads to low individual mortality in places where local plant cover is high. We will refer to this feature of a spatially constrained negative density dependence of mortality as 'associational resistance' (see Milchunas and Noy-Meir 2002 for a discussion on the term). Vice versa, a low local plant cover will increase the risk of dying due to grazing for the remaining vegetation (Milchunas and Noy-Meir 2002). As a consequence, if the overall benefit of grazing protection outweighs the cost of competition for limiting resources, plants coincidentally team up with other plants (Atsatt and O'Dowd 1976; Graff et al. 2007), which contributes to the formation of patch pattern at the landscape scale (Sala 1988; Milchunas and Noy-Meir 2002). In such case, the highest risk of being grazed is borne by plants that grow isolated from others, whereas plants that are growing at the border or in the center of a patch are less vulnerable to grazing or even entirely unaffected because they benefit from associational resistance (Milchunas and Noy-Meir 2002; Barbosa et al. 2009).

Here, we introduce associational resistance in a spatially explicit dryland vegetation model to investigate the effect of a heterogeneous pressure on pattern formation and ecosystem resilience. We hypothesize that such spatially heterogeneous pressure adds positive feedbacks to the process of patch formation, which may as a consequence reinforce the emergence of sharp thresholds for ecosystem degradation. Furthermore, because the emerging spatial structure is affected by the pressure, it is worthwhile investigating the behavior of the suggested spatial indicators of degradation: how do the spatial indicators, and the patch size distribution in particular, behave under a spatially heterogeneous pressure? 


\section{Methods}

We introduced the mechanism of associational resistance by which neighboring plants protect each other from grazing in a model of dryland vegetation dynamics (Kéfi et al. 2007b). The model is an interaction particle system (Durrett and Levin 1994) that describes the landscape as a grid of cells, each of which can be in one of three states: 'vegetated' cells are occupied by a plant (annotated as ' + ' in equations; black cells in figures); 'empty' cells do not contain adult plants but are suitable for seeds to germinate and establish ('0', grey cells); 'degraded' cells represent bare ground which has been eroded, lacks organic matter, is characterized by bad water retention, and therefore cannot be colonized by arriving seeds ('-', white cells).

Transitions between cell states are only possible between vegetated and empty (by the processes of plant 'death' and 'recolonization') and between empty and degraded (by 'degradation' and 'regeneration'). In biological terms, a degraded site needs to be enriched first, before a plant can establish on it. Conversely, when a plant dies, it leaves the spot empty but still enriched, until it becomes degraded by erosion. The probabilities for these transitions to occur on any cell currently at state $i$ are defined in the following paragraphs. They might be constants or functions of the global vegetation cover, $\rho_{+}$, or of the local vegetation cover in the neighborhood of the focal cell, $q_{+\mid i}$ (i.e. proportion of neighbors in state ' + ' given that the focal cell is in state ' $i$ ').

\section{The model}

Under the harsh environmental conditions of drylands, plants enhance the abiotic conditions in their direct neighborhood by accumulating organic matter, providing shade and retaining water. The original model by Kéfi et al. (2007b) mimics such local facilitation of plants in drylands by defining the probability $w_{\{-, 0\}}$ of a degraded cell (state '-') to regenerate (change into state ' 0 ') as a function of the plant cover in the direct neighborhood, $q_{+\mid-}$(four nearest cells, i.e. "von Neumann"-neighborhood of range 1, Kéfi et al. 2007b):

$w_{\{-, 0\}}=r+q_{+\mid-} f$,

where $r$ is the intrinsic regeneration rate of degraded cells in the absence of vegetated neighbors, and $f$ is the intensity of facilitation provided by vegetated neighbors, which is maximal when all four neighbors are occupied (i.e. $q_{+\mid-}=1$ ). Thus, plants act as 'ecosystem engineers' that increase the availability of their own habitat (Gurney and Lawton 1996; Jones et al. 1997; Gilad et al. 2007; Hastings et al. 2007).
The probability of empty cells (in state ' 0 ') to be recolonized by vegetation is

$w_{\{0,+\}}=\left(\delta \rho_{+}+(1-\delta) q_{+\mid 0}\right)\left(b-c \rho_{+}\right)$,

where the first term of the equation represents seed dispersal including the proportion, $\delta$, of seeds originating from all over the lattice (global dispersal), and the proportion $1-\delta$ of seeds arriving from plants in the direct neighborhood (local dispersal). The second term represents the germination and early survival rate, $b$, in the absence of competition which decreases with the global competition for limited resources, $c$, reflecting that the recruitment of a new plant becomes more difficult with increasing plant cover, $\rho_{+}$, because of competition for the limited resource at the landscape scale (Kéfi et al. 2007b). The germination and early survival rate, $b$, is a direct consequence of the environmental quality of the landscape and therefore we vary this term as a proxy for homogeneous environmental pressure.

The probability of empty cells to degrade is a constant rate, $d$ :

$w_{\{0,-\}}=d$.

Finally, in the original model of Kéfi et al. (2007b), the intrinsic plant mortality rate is defined as a constant, $w_{\{+, 0\}}=m_{0}$, which can be interpreted as the inverse of the average plant lifespan. While this approach assumed spatially homogeneous pressure (Fig. 1a), associational resistance renders grazing pressure on an individual plant dependent on its local neighborhood and therefore on the current spatial configuration (Fig. 1b).

To account for the spatially heterogeneous impacts of grazing due to associational resistance, we assumed that a plant's vulnerability to grazers decreases with the proportion of occupied neighbors, $q_{+\mid+}$. The individual probability of dying is therefore defined as

$w_{\{+, 0\}}=m_{0}+g_{0}\left(1-q_{+\mid+}\right)$,

where the additional mortality due to grazing is maximized to $g_{0}$ if a plant has no vegetated neighbor (i.e. $q_{+++}=0$ ) and gradually reduces to 0 with an increasing fraction of occupied neighbors, $q_{+\mid+}$.

\section{Numerical simulations}

We applied the transition probabilities defined in Eqs. 1-4 on $100 \times 100$ squared grid cells assuming that each grid cell covers an area of about $0.25 \mathrm{~m}^{2}$ (the average size of an adult plant individual). We initiated the landscape with two different starting conditions: from high cover to quantify vegetation patterns in an undegraded landscape and from very low cover to assess the ability of the landscape to recover 
Fig. 1 Individual plant risk of death due to grazing (bars). a In most previous spatially explicit models of dryland resilience, grazing was defined homogeneously, i.e. affected all plants likewise, regardless of local cover. b A more realistic approach to grazing assumes plant death to be reduced by the presence of other plants in the direct neighborhood, i.e. the mechanism of 'associational resistance', with implications for the total plant mortality at both high (left panels) and low (right panels) vegetation cover

a) homogenous grazing pressure
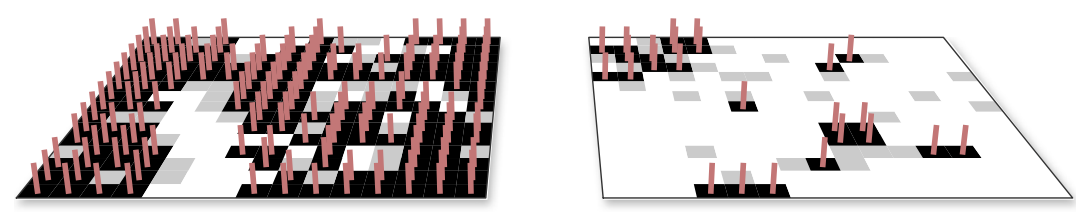

b) spatially heterogeneous grazing pressure

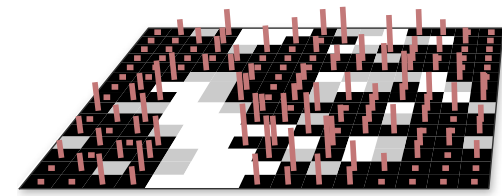

high vegetation cover

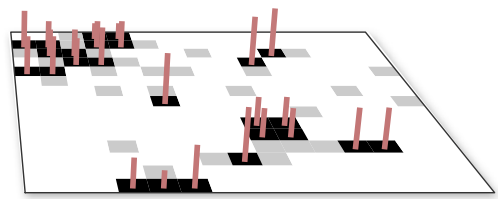

low vegetation cover

environmental pressure

from a degraded state (see below for further specifications). A timestep was defined to include the dynamics occurring within 1 year. The model dynamics followed stochastic processes: at each timestep, the transition probabilities of each cell were compared against a uniform random number to determine if the cell remained unchanged or if one of the possible transitions occurred (i.e. synchronous updating). The local densities were calculated assuming periodic boundary conditions (i.e. cells at left/upper border were neighboring the cells at the right/lower border and vice versa).

The model of this study inherited all parameters from the model of Kéfi et al. (2007b; $r=0.01, f=0.9, \delta=0.1$, $c=0.2, d=0.1)$. We assumed only a small proportion of long-range dispersal, $\delta$, following the empirical evidence for predominantly local dispersal in drylands (Aguiar and Sala 1994). Furthermore, the intrinsic mortality $\left(m_{0}=0.05\right)$ and grazing intensities $\left(0<g_{0}<0.5\right)$ reflected an average individual lifespan between 20 years, if no additional grazing mortality was taking effect due to associational resistance, and 2 years for strongly exposed plants (Condit et al. 1995).

If not stated otherwise, we performed the following simulations and measurements along a gradient of grazing intensity, $g_{0}$, and environmental pressure, $(1-b)$.

\section{Quantifying the vegetation patterns}

The state of persistent vegetation cover was assessed by starting grids from randomly scattered high plant cover with $0.8 \leq \rho_{+} \leq 0.9$ and $50 \%$ of the remaining cells in the degraded state. Dynamics were run until a steady state was reached (i.e. difference of mean vegetation cover, $\rho_{+}$, over two subsequent periods of 200 years was inferior to $10^{-6}$ ) or the vegetation went extinct. The simulations were replicated 5-10 times. Replicates that did not reach a steady state after 5000 years were discarded (less than $1 \%$ of total simulation runs), but simulations were repeated until at least five replicates fulfilled the criterion.

A set of simple descriptors of the landscape state were calculated as the average in a period of the final 400 years of the simulation after steady state. These included the average total vegetation cover, $\rho_{+}$, the average local vegetation cover, $\overline{q_{+1+}}$ (i.e. the cover in the local neighborhood, $q_{+1+}$, averaged over all vegetated cells on the grid) and the clustering coefficient, $c_{++}=\rho_{+} / \overline{q_{+\mid+}}$, which is larger than one if plants are more associated in space than expected by chance.

Additionally, more advanced metrics of spatial structure were assessed at the landscape scale at the end of the simulations. For each replicate, the number and size of vegetation patches, i.e. continuously vegetated areas connected by at least one side of a cell, were assessed. Of these, the largest patch size, $\overline{s_{\max }}$, was calculated and averaged across all replicates of a parameter set.

Furthermore, the inverse cumulative distribution of patch sizes observed in the simulated landscapes was derived: unique patch sizes, $s$, occurring at the end of each simulation run were ranked and for each value the frequency, $p$, of a given patch being equal or larger than $s$ was calculated by dividing $s$ by the total number of patches in the landscape (White et al. 2008). We pooled the inverse cumulative patch-size distributions (i.e. the obtained $p$ and $s$ values) of all replicates for a given parameter set $(n=5-10)$ before fitting models to these distributions. Assessing the shape of the inverse cumulative patch-size distribution was done in two steps: To test for up-bent vs. down-bent curvature of the distributions, we fitted a polynomial model 
on bootstrapped data $\left(\log (p)=a \log (s)+b \log (s)^{2}\right.$; ordinary nonparametric resampling using the R-package 'boot'; Canty and Ripley (2015); least-squares fitting) to obtain $95 \%$ confidence intervals of the parameters $a$ and $b$ of the polynomial. We then chose one of the following probability density functions based on the significance and algebraic sign of the parameter $b$, which determines the curvature of the fitted polynomial (Kéfi et al. 2007a, 2014; White et al. 2008): a pure power law ( $\left.p=s^{-\lambda+1}\right)$ if no significant curvature was detected, i.e. the confidence intervals for $b$ included zero ( $\lambda$ corresponds to the exponent of the correponding non-cumulative patch-size distribution); an up-bent power law due to the presence of large spanning patches in the landscape, ranging from one edge of the lattice to another $\left(p=p_{\text {min }}+s^{-\lambda+1} ; p_{\text {min }}\right.$ being the lower limit of observed probabilities) if confidence intervals for $b$ only included positive values; and a down-bent power law with a truncation threshold $\left(p=s^{-\lambda+1} \exp \left(s / s_{\max }\right)\right)$ if confidence intervals for $b$ only included negative values (the model selection by bootstrapped polynomial coefficients is respecting the error structure of the data and is more conservative than model selection using AIC). The log-log transforms of these models were fitted using nonlinear leastsquares on the log-log-transformed data (White et al. 2008). This method is adequatly mapping the shape of the distributions (other fitting methods, e.g. maximum-likelihood estimation, would have been marginally more precise but were unavailable for the up-bent power-law function, White et al. 2008; Clauset et al. 2009).

Eventually, we assigned the outcome of the simulations obtained for each parameter set to one of the following five cases: (i) fully vegetated, if the vegetation cover was almost complete $\left(\rho_{+}>0.8\right)$ and thus largely aggregated into one single patch; (ii) up-bent power law, if the inverse cumulative patch-size distribution was best fitted by a power law with a lower limit defined by large spanning patches; (iii) power law, if the inverse cumulative patch-size distribution was best described by a straight power law; (iv) down-bent power law, if the inverse cumulative patch-size distribution was best described by a truncated power law where the large vegetation patches were significantly smaller than expected in a pure power law; (v) desert, if the vegetation cover was negligible $\left(\rho_{+}<0.01\right)$. We compared the exponent $\lambda$ of the power law for the cases ii-iv.

\section{Capacity of the ecosystem to recover}

In a second set of simulations, we ran the same parameter combinations but starting from a desert state in which a very low initial vegetation cover was introduced (randomly scattered plants, $\rho_{+}=0.001$, in a fully degraded landscape, $\rho_{-}=0.999$; i.e. a 'perturbation' of the desert state by adding ten vegetated cells). The simulations started with
1 year of regeneration only before grazing set in, and were run until vegetation went extinct or reached a vegetation cover of $1 \%$ within a period of 100 years (corresponding to a tenfold increase in initial vegetation), in which case we considered that the landscape was undergoing recovery. Since the events in the model are stochastic, not every replicate would recover with certainty, even if the conditions are feasible. Therefore, those simulations were replicated 100 times. Note also that the size of the lattice constrains the minimal possible plant cover $\left(\min \left(\rho_{+}\right)=0.0001=\right.$ $1 /(100 \times 100))$.

We considered the degradation to be irreversible, i.e. the desert state being 'stable', if less than $50 \%$ of the replicates recovered. Conversely, we considered the degradation to be reversible if more than $50 \%$ of the replicates recovered.

\section{Results}

Simultaneously increasing environmental $(1-b)$ and grazing $(g)$ pressures showed a gradual decline in vegetation cover (Fig. 2a; black contours) until it reached a threshold value of pressure after which the landscape degraded into a desert (which we defined as a landscape with less than $1 \%$ cover). Subsequently, we refer to this threshold value as the 'tipping point'. Once degraded, restoration was unlikely (gray area in Fig. 2a) unless the combined pressure level was decreased below a second threshold (border between gray and white areas). The pressure levels where both desert and vegetated landscapes co-exist (i.e. overlap of grey area and black contours in Fig. 2a) define the domain of 'bistability'. In this range of pressure levels, the ecosystem can be in either one of the two states depending on its history, and perturbations can push the ecosystem from one stable state to the other. The bistability domain became wider with increasing grazing pressure, indicating that grazing reduced the ecosystem resilience (Fig. 2a).

For clarification, we show two cross-sections through the parameter space at low vs. high grazing pressure $\left(g_{0}=\right.$ 0.1 vs. $g_{0}=0.4$, Fig. $\left.2 \mathrm{~b}, \mathrm{c}\right)$. In both cases, vegetation cover declined with increasing environmental pressure until it reached a tipping point at which the ecosystem degraded into a desert. When grazing pressure was high, vegetation cover was still high at the tipping point $\left(\rho_{+}=0.44\right.$; Fig. $2 b$, upper line). Moreover, once degraded to a desert, vegetation was unlikely to recover since the desert state was stable even if the environmental conditions were improved (lower line). In contrast, at low grazing level, the tipping point occurred at much higher levels of environmental pressure and the vegetation cover of the landscape was low before collapsing to a desert $\left(\rho_{+}=0.16\right)$, meaning that low vegetation cover could be sustained in the ecosystem (Fig. 2c, upper line). Also, once in a desert state, a reduction of the environmental 
a)

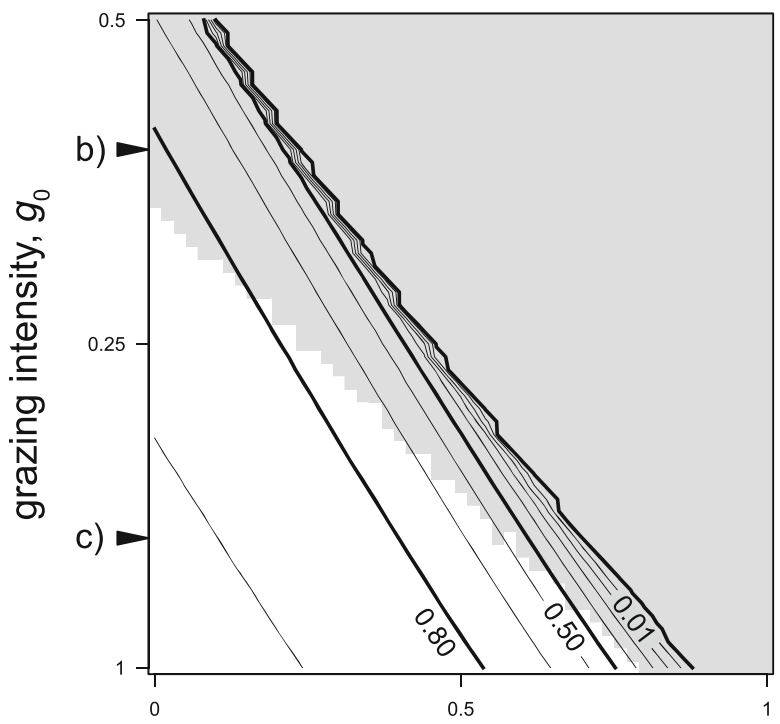

environmental pressure, (1-b)

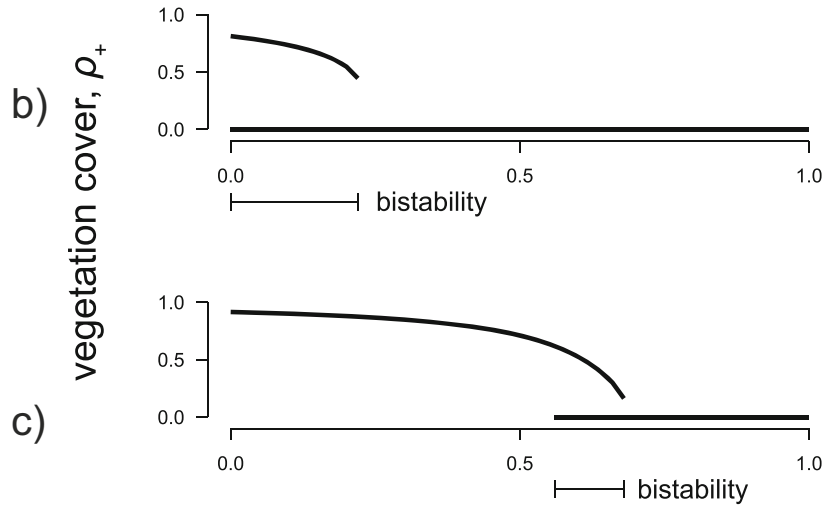

environmental pressure, $(1-b)$

Fig. 2 a Changes in the vegetated (black contour lines: $\rho_{+}$) and desert (i.e. no vegetation) states (grey zone) along gradients of environmental and grazing pressure; overlap of the grey zone and the contour lines correspond to the bistability area where both the vegetation and the desert states are stable. The tipping point, at which the ecosystem drops from vegetated to desert, is reached at the lowest contour line $\left(\rho_{+}<\right.$ 0.01). (b, c) Cross sections of (a) at low (b; $\left.g_{0}=0.1\right)$ and high (c; $g_{0}=0.4$ ) grazing intensities showing the steady state vegetation cover

pressure would improve the probability for a recovery of the ecosystem (Fig. 2c, lower line).

\section{Spatial indicators}

We used the shape of the inverse cumulative patch-size distributions to assign the simulated landscapes in categories (see methods) distinguishing landscapes of highly connected vegetation cover (Fig. 3(i and ii), black and grey) from landscapes where large vegetation patches were overproportionally fragmented (marked by down-bent, truncated power laws; Fig. 3(iv), red). A straight power law (Fig. 3(iii), orange) describes the margin between these two cases. The same sequence of vegetation patterns $(\mathrm{i}-\mathrm{v})$ was observed along both gradients of pressures. However, the pressure levels at which truncated power-laws (iv in red) were observed became more constrained as grazing pressure increased, until they disappeared at the highest grazing levels. In those cases, the tipping point at which vegetation was suddenly lost was not preceded by truncated power-laws, but happened in landscapes were vegetation patches were best described by straight power-laws (Fig. 3).

To gain further understanding in the response of the spatial patterns to increasing pressure levels, we tracked the average largest patch size $\overline{s_{\max }}$, the clustering coefficient $c_{++}$and the exponent of the inverse cumulative patch-size distribution $\lambda$ along the two pressure gradients. When observing along a gradient of environmental pressure, regardless of the grazing level, the largest patch size (Fig. 4a-c) dropped as the inverse cumulative patch-size distribution approached the straight power-law distribution. At low grazing, the largest patch size dropped dramatically from covering approximately $60 \%$ of the entire landscape to significantly smaller patches covering less than $20 \%$ before collapsing into a desert. The clustering coefficient (Fig. $4 \mathrm{~d}-\mathrm{f}$ ) increased with environmental pressure, but the values reached just before the collapse were much higher at low grazing level than at high grazing level. The powerlaw exponent, $\lambda$ (Fig. 4g-i), the parameter that describes the change in patch frequency with patch size for the smaller patches, was constantly declining (from approx. 3.5 to 1.7 ) along the environmental pressure gradient.

Thus, as environmental and grazing pressure increased, the spatial metrics of vegetation seemed to respond qualitatively in a consistent way, which suggests that they may contribute to building monitoring tools of ecosystem degradation. However, at high grazing levels, there were clear signs of a qualitative alteration of the spatial structure close to the threshold to degradation. More precisely, at high grazing levels, the vegetation patterns were characterized by a power law were characterized by a power law, and not by a truncated power-law, just before the ecosystem degradation to desertification. More generally, the trends and maximum values of all spatial metrics were weaker under high than under low grazing pressure (Fig. 3). Thus, the interpretation of the spatial patterns, and hence their potential role as indicators, depends on the level of grazing pressure in this system.

\section{Discussion}

A number of spatially explicit ecosystem models have contributed to predicting the risk of catastrophic shifts using early warning signals (e.g. Rietkerk et al. 2004; Kéfi et al. 2007b, 2014; Guttal and Jayaprakash 2009; 


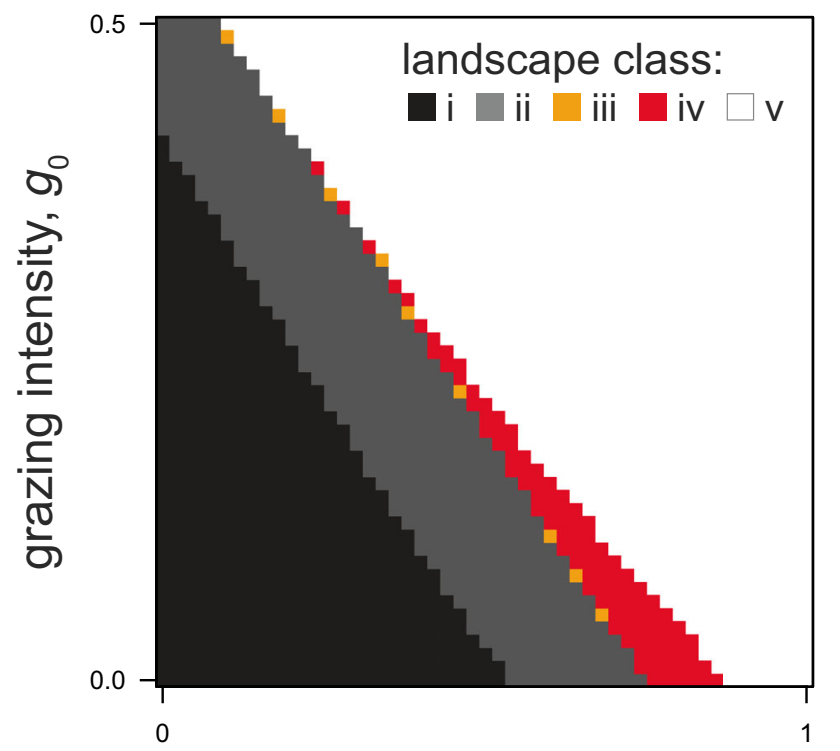

environmental pressure, $(1-b)$
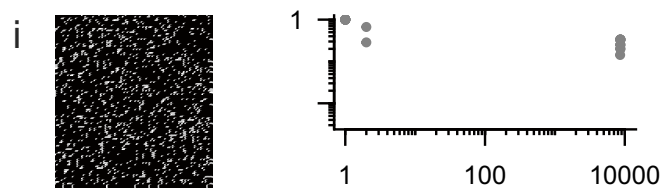

ii

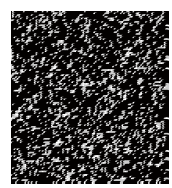

iii
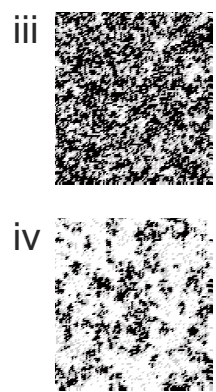

V
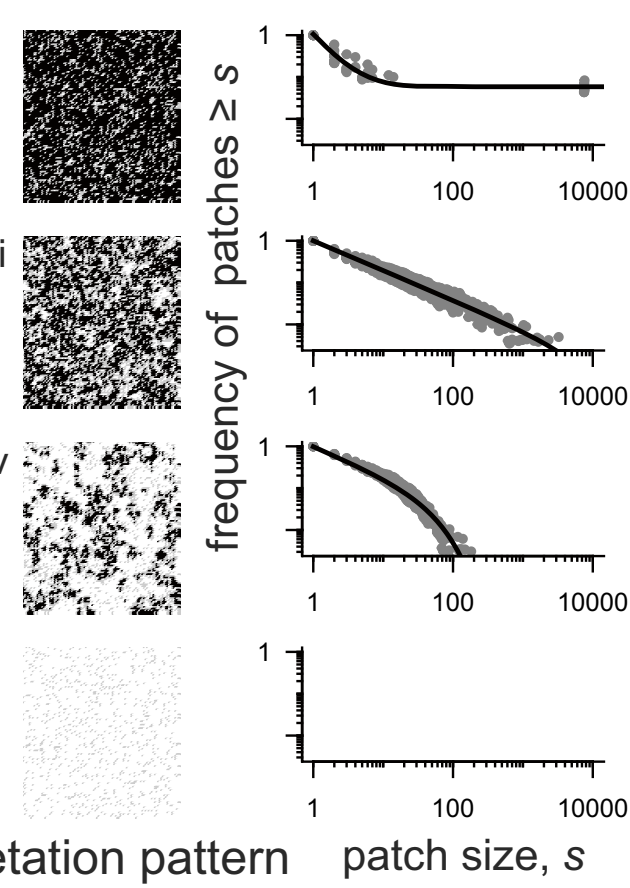

Fig. 3 Landscapes classified based on the shape of the inverse cumulative patch-size distributions obtained from pooled data of $n$ independent replicates along gradients of environmental and grazing pressures (where $n$ is between five and ten replicates for each combination of environmental and grazing pressures). Classes are $i$ : full cover; $i i$ : up-bent power-law with spanning clusters; $i i i$ : straight power-law; $i v$ : down-bent power law; $v$ : desert. Note that at high grazing pressure, a vegetation collapse was not preceded by down-bent power laws (iv). If crossing the tipping point, the system transitions from vegetated (i.e. colored area) to desert (white area)
Dakos et al. 2010). Until now, these models have not investigated the possible spatial component of external pressures. In this study, we show that spatially explicit pressures may fundamentally alter the resilience properties of ecosystems and that it is important to take the interactive character of the feedback mechanisms behind catastrophic shifts into account.

As an illustration, we studied a mechanistic model of vegetation dynamics in grazed drylands in which we introduced the mechanisms of plants' 'associational resistance'-i.e. the mutual protection from grazing of plants growing next to each other. We used this model to investigate resilience as well as the emergent spatial patterns, which have been proposed as candidate early warning signals of catastrophic shifts in drylands (Rietkerk et al. 2004; Kéfi et al. 2007a).

Our model analyses suggest that a spatially explicit pressure can interfere with other spatially explicit mechanisms, such as plant-soil feedbacks, and may thereby alter the relationship between spatial patterns and ecosystem resilience. First, the range of pressure levels at which both desert and vegetated landscapes were simultaneously stable (i.e. the socalled bistability area) increased as spatially explicit grazing became more intense. This is consistent with previous modelling studies of spatially homogeneous grazing pressure (Rietkerk et al. 2002; Kéfi et al. 2007a, 2007b). Second, under high grazing pressure, state transitions from a vegetated to a bare landscape were more sudden and unexpected. The ecosystem shift to desert occurred at higher vegetation cover and connectivity, i.e. in apparently 'healthy' landscapes, and became more difficult to predict using spatial indicators.

\section{Two pressures, one shift}

It is important to highlight that the interference of grazing and environmental pressure was a property that emerged at the patch scale from the feedback mechanisms on mortality and recruitment defined at the plant individual level. On the one hand, environmental pressure affected plant seedling establishment and early survival. That process depended on the local facilitation mechanism which favored the regeneration of degraded sites in the neighborhood of already established plants. On the other hand, grazing affected the mortality of mature plants. That depended on the mechanism of associational resistance which decreases the risk of being consumed when in the neighborhood of other established plants. Thus, at any given time, the two mechanisms acted on different individuals, in different life history stages and at different locations in the landscape. Still, over the course of time, these mechanisms affected vegetation patches by determining growth and mortality at the edge of the patches linked only through the spatially explicit 
a) largest patch size

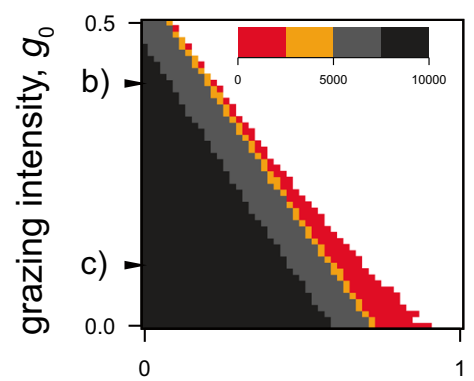

b)

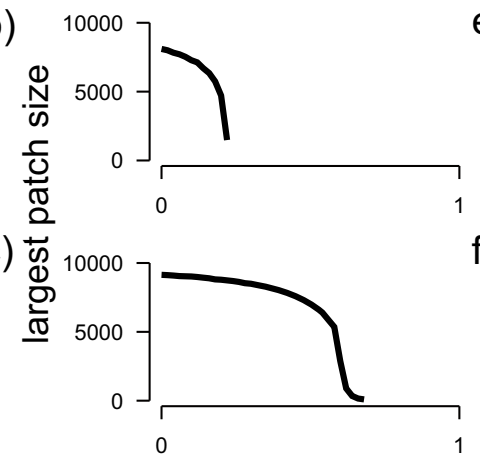

d)
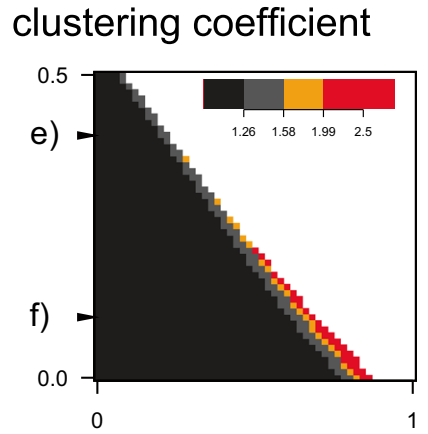

e)

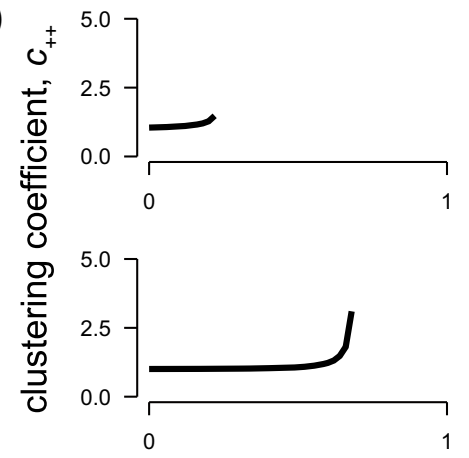

g) exponent, $\lambda$

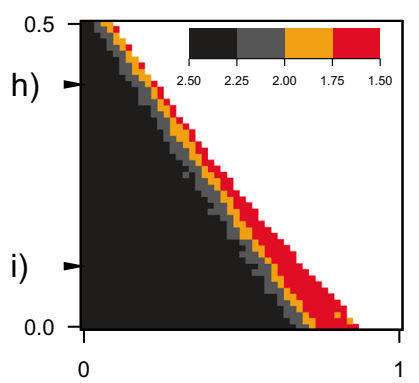

h)

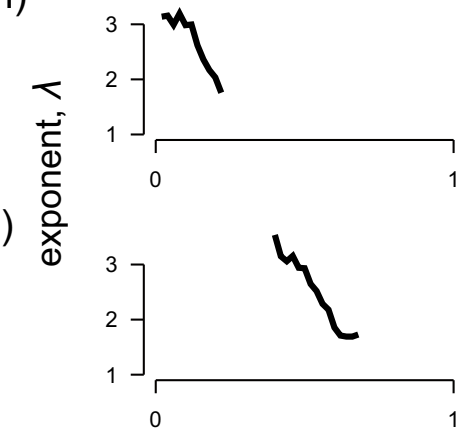

environmental pressure, $(1-b)$

Fig. 4 Spatial metrics along gradients of grazing and environmental pressures. a-c Largest patch size $\overline{s_{\max }}, \mathbf{d}-\mathbf{f}$ clustering coefficient $c_{++}$ and $\mathbf{g}-\mathbf{i}$ exponent $\lambda$ of the fitted probability density functions. Second

'memory' of the landscape. In other words, patches were shaped by grazing and aridity in highly complex, interactive ways that are difficult to disentangle quantitatively. The two pressures were thus coupled by cross-scale interactions in space and time to define a single threshold for a catastrophic shift at the ecosystem level.

\section{Spatial indicators of degradation}

Metrics of spatial patterns, such as the clustering coefficient and the patch size distributions, have attracted a lot of attention in the literature as potential early warning signs or indicators of ecosystem degradation (Kéfi et al. 2007b; Maestre et al. 2009; Lin et al. 2010). Given that such spatial metrics are relatively easy to assess using image processing, air-borne imagery or remote sensing (Fuentes et al. 1984; Barbier et al. 2006; Scanlon et al. 2007; Deblauwe et al. 2008), understanding their ability to predict degradation would provide a useful tool for sustainable land use management.

At low grazing pressure, the sequence of spatial patterns along a stress gradient observed in our model is in agreement with previous models assuming spatially homogeneous pressure (Kéfi et al. 2007b; Manor and Shnerb 2008; von Hardenberg et al. 2010) and empirical studies (Kéfi et al. 2007b; Lin et al. 2010): with increasing pressure, and third rows correspond to cross-sections of the first row at high ( $g=0.4)$ and low $(g=0.1)$ grazing level, respectively

spanning vegetation clusters were replaced by power-law distributions, which disintegrated into truncated power-laws before the collapse of the landscape. It is noteworthy that vegetation cover decreased along gradients of environmental pressures and reached low levels before the ecosystem collapsed (therefore low vegetation cover landscapes were sustainable at low grazing). However, the results presented here show that this scenario does not operate under high grazing pressure. Then, the degradation risk can be high even in landscapes showing straight power-law distributions since a strong spatially explicit feedback may delay the disintegration of patches. Indeed, as grazing pressure increases, the positive feedback induced by associational resistance cumulatively adds to the local facilitation mechanism to promote plant clustering and the formation of large patches. More specifically, the threshold of ecosystem collapse coincided with power-law distributions of patch-sizes. This means that even landscapes exhibiting high total vegetation cover and pure power-law patch size distributions were prone to degradation under high grazing pressure. Under high grazing pressure, the interpretation of the observed vegetation patterns is altered and the early warning signals of degradation change from truncated power law to power law.

Our results suggest that knowledge about the possible spatial components of feedback mechanisms is of great 
importance to understand pattern formation and their interpretation (Fuentes et al. 1984; Aguiar and Sala 1999). The model proposed here is just a first step towards a higher degree of realism in spatially explicit models of resilience. We anticipate that models that aim to predict pressure thresholds for applied ecosystem management (Westoby et al. 1989; Suding and Hobbs 2009) will need to identify and include the relevant sources of heterogeneity as well as their spatial scales in the positive feedback mechanisms.

Note that in the present study, we investiagte the spatial patterns at steady state for each level of a given environmental condition. Going along a gradient of the environmental conditions, this assumes that the ecosystem has the time to reach steady state before the environmental conditions changes, i.e. that the change in the external condition is slower than the ecological dynamics of the system. In the case where the changes in external conditions would occur at similar or faster pace than the ecological dynamics, the behavior of the spatial patterns, and their sequence of changes along the gradient would deserve further investigations.

\section{Positive feedbacks and criticality}

Pascual and Guichard (2005) have highlighted how different disturbance and recovery mechanisms lead to different types of criticality and thereby different spatial signatures. In our model without associational resistance, all plants have the same probability of being consumed by grazers independent of their local spatial configuration (i.e. whether they have neighbors or not). Grazing has no spatial component in this case. Vegetation recovery by recruitment, however, is rendered spatially explicit through the local facilitation mechanism (a local recovery process, Pascual and Guichard 2005). Previous work (Kéfi et al. 2011) has shown that this system exhibits properties similar to 'robust criticality' (sensu Pascual and Guichard 2005; such as found in mussel beds, Pascual et al. 2002; Guichard et al. 2003; Roy et al. 2003), and power-law scaling in this case occurs at the percolation point without being associated with an abrupt change in the vegetation cover of the landscape. The patch size distribution increasingly deviates from a pure power law as the system approaches the extinction threshold of vegetation cover below which desertification is inevitable (i.e. a catastrophic shift; Kéfi et al. 2011).

When associational resistance is added to this model, as proposed in the present study, grazing intensity becomes dependent on the local plant density (a 'well-mixed' or 'distributed' disturbance sensu Pascual and Guichard 2005). Local plant mortality is highest if plants have few or no neighbors. Consequently, fragmented vegetation patches experience a higher pressure and loss of plant individuals than aggregated patches. This type of disturbance has been suggested to favor 'classical criticality' which is associated with scale invariance at the critical point (e.g. as observed in wind disturbed tropical forest, Kizaki and Katori 1999, Pascual and Guichard 2005). Thus, our model combines local recovery with a well-mixed disturbance. Following the classification of Pascual and Guichard (2005), as the intensity of grazing pressure increases (i.e. the disturbance), so does the mechanism favoring classical criticality, suggesting that the system moves from robust to classical criticality along the grazing gradient. The introduction of a spatially heterogeneous disturbance intimately interferes with both the pattern formation and the ecosystem resilience, which are tightly linked in those ecosystems. As a consequence, the interpretation of the patterns changes as well. While in robust critical systems power laws indicate that the system is still relatively resilient, in classic critical systems power laws indicate that the system is at (or very close to) the critical point (Pascual and Guichard 2005; Kéfi et al. 2011).

\section{Conclusion}

Our results indicate that when ignoring the interfering feedback mechanisms caused by spatially explicit pressure, we might over-estimate ecosystem resilience and impede the success of sustainable management practices. To understand sudden degradation, we must develop more integrative views that extrapolate from spatially heterogeneous feedback mechanisms occurring at the local scale to spatial patterns and resilience at the landscape scale. In the case example of drylands under livestock grazing pressure, this means that we must incorporate spatially explicit plant mortality due to grazing into our models to see if early warning signs of spatial structure do apply under the given circumstances. More generally, our study warns about the possible effect of spatially heterogeneous pressures on spatial metrics since they may interact with the mechanisms responsible for pattern formation. Thereby, spatially explicit pressures may alter the qualification of spatial metrics for use as 'early-warning signs' of degradation. We conclude that the identification of the main external pressures involved in pattern formation is a prerequisite for the development of reliable spatial indicators of catastrophic shifts.

Acknowledgments We thank four anonymous reviewers for their useful comments on the manuscript and François Rousset for suggestions on model comparison and model fitting.

Author contributions FDS developed the simulation code. FDS and SK conceived the study, analysed the simulation results and wrote the manuscript. All authors gave final approval for publication.

Source code repository We commit ourselves to a transparent and reproducible research and made the documented source code that was used for the simulations available on GitHub (simulations were 
performed in R; https://cascade-wp6.github.com/2015_schneider_kefi; doi:10.5281/zenodo.35034).

\section{Compliance with Ethical Standards}

Funding The research leading to these results has received funding from the European Union Seventh Framework Programme (FP7/20072013) under grant agreement no. 283068 (CASCADE project). This is publication ISEM 2015-264. The authors declare that they have no conflict of interest.

Open Access This article is distributed under the terms of the Creative Commons Attribution 4.0 International License (http:// creativecommons.org/licenses/by/4.0/), which permits unrestricted use, distribution, and reproduction in any medium, provided you give appropriate credit to the original author(s) and the source, provide a link to the Creative Commons license, and indicate if changes were made.

\section{References}

Aguiar MR, Sala OE (1999) Patch structure, dynamics and implications for the functioning of arid ecosystems. Trends Ecol Evol 14:273-277. doi:10.1016/S0169-5347(99)01612-2

Aguiar MR, Sala OE (1994) Competition, facilitation, seed distribution and the origin of patches in a patagonian steppe. Oikos 70:26-34. doi:10.2307/3545695

Asner GP, Elmore AJ, Olander LP et al (2004) Grazing systems, ecosystem responses, and global change. Annu Rev Environ Resour 29:261-299. doi:10.1146/annurev.energy.29.062403.102142

Atsatt PR, O'Dowd DJ (1976) Plant defense guilds. Science 193:2429. doi:10.1126/science.193.4247.24

Bailey DW, Gross JE, Laca EA et al (1996) Mechanisms that result in large herbivore grazing distribution patterns. J Range Manag 49:386-400. doi: $10.2307 / 4002919$

Bailey RM (2011) Spatial and temporal signatures of fragility and threshold proximity in modelled semi-arid vegetation. Proc R Soc Lond B Biol Sci 278:1064-1071. doi:10.1098/rspb.2010.1750

Baraza E, Zamora R, Hódar JA (2006) Conditional outcomes in plan therbivore interactions: neighbours matter. Oikos 113:148156. doi:10.1111/j.0030-1299.2006.14265.x

Barbier N, Couteron P, Lejoly J et al (2006) Self-organized vegetation patterning as a fingerprint of climate and human impact on semi-arid ecosystems. J Ecol 94:537-547. doi:10.1111/j.1365-2745.2006.01126.x

Barbosa P, Hines J, Kaplan I et al (2009) Associational resistance and associational susceptibility: having right or wrong neighbors. Annu Rev Ecol Evol Syst 40:1-20. doi:10.1146/annurev.ecolsys.110308.120242

Borgogno F, D'Odorico P, Laio F, Ridolfi L (2009) Mathematical models of vegetation pattern formation in ecohydrology. Rev Geophys 47:RG1005. doi:10.1029/2007RG000256

Callaway RM (1995) Positive interactions among plants. Bot Rev 61:306-349. doi:10.1007/BF02912621

Canty A, Ripley BD (2015) Boot: Bootstrap r (S-Plus) functions

Carpenter SR, Cole JJ, Pace ML et al (2011) Early warnings of regime shifts: a whole-ecosystem experiment. Science 332:1079-1082. doi:10.1126/science. 1203672

Clauset A, Shalizi CR, Newman ME (2009) Power-law distributions in empirical data. SIAM Rev 51:661-703. doi:10.1137/070710111

Cline TJ, Seekell DA, Carpenter SR et al (2014) Early warnings of regime shifts: evaluation of spatial indicators from a whole-ecosystem experiment. Ecosphere 5:art102. doi:10.1890/ES13-00398.1
Condit R, Hubbell SP, Foster RB (1995) Mortality rates of 205 neotropical tree and shrub species and the impact of a severe drought. Ecol Monogr 65:419-439. doi:10.2307/2963497

Dakos V, Carpenter SR, Brock WA et al (2012) Methods for detecting early warnings of critical transitions in time series illustrated using simulated ecological data. PLoS ONE 7:e41010. doi:10.1371/ journal.pone.0041010

Dakos V, van Nes EH, Donangelo R et al (2010) Spatial correlation as leading indicator of catastrophic shifts. Theor Ecol 3:163-174. doi:10.1007/s12080-009-0060-6

Deblauwe V, Barbier N, Couteron P et al (2008) The global biogeography of semi-arid periodic vegetation patterns. Glob Ecol Biogeogr 17:715-723. doi:10.1111/j.1466-8238.2008.00413.x

Díaz S, Lavorel S, McIntyre S et al (2007) Plant trait responses to grazing a global synthesis. Glob Chang Biol 13:313-341. doi:10.1111/j.1365-2486.2006.01288.x

Durrett R, Levin S (1994) The importance of being discrete (and spatial). Theor Popul Biol 46:363-394. doi:10.1006/tpbi.1994.1032

Fuentes ER, Otaiza RD, Alliende MC et al (1984) Shrub clumps of the Chilean matorral vegetation: structure and possible maintenance mechanisms. Oecologia 62:405-411. doi:10.1007/BF00384275

Gilad E, von Hardenberg J, Provenzale A et al (2007) A mathematical model of plants as ecosystem engineers. J Theor Biol 244:680 691. doi:10.1016/j.jtbi.2006.08.006

Graff P, Aguiar MR, Chaneton EJ (2007) Shifts in positive and negative plant interactions along a grazing intensity gradient. Ecology 88:188-199. doi:10.1890/0012-9658(2007)88[188:SIPANP]2.0.CO;2

Guichard F, Halpin PM, Allison GW et al (2003) Mussel disturbance dynamics: signatures of oceanographic forcing from local interactions. Am Nat 161:889-904. doi:10.1086/375300

Gunderson LH (2000) Ecological resilience in theory and application. Annu Rev Ecol Syst 425-439. doi:10.2307/221739

Gurney WSC, Lawton JH (1996) The population dynamics of ecosystem engineers. Oikos 273-283. doi: $10.2307 / 3546200$

Guttal V, Jayaprakash C (2009) Spatial variance and spatial skewness: leading indicators of regime shifts in spatial ecological systems. Theor Ecol 2:3-12. doi:10.1007/s12080-008-0033-1

Hastings A, Byers JE, Crooks JA et al (2007) Ecosystem engineering in space and time. Ecol Lett 10:153-164. doi:10.1111/j.1461-0248.2006.00997.x

Holling CS (1973) Resilience and stability of ecological systems. Annu Rev Ecol Syst 4:1-23. doi:10.1146/annurev.es.04.110173.000245

Jones CG, Lawton JH, Shachak M (1997) Positive and negative effects of organisms as physical ecosystem engineers. Ecology 78:1946-1957. doi:10.1890/0012-9658(1997)078[1946:PANEOO]2.0.CO;2

Kéfi S, Guttal V, Brock WA et al (2014) Early warning signals of ecological transitions: methods for spatial patterns. PloS one 9:e92097. doi:10.1371/journal.pone.0092097

Kéfi S, Rietkerk M, Alados CL et al (2007a) Spatial vegetation patterns and imminent desertification in Mediterranean arid ecosystems. Nature 449:213-217. doi:10.1038/nature06111

Kéfi S, Rietkerk M, van Baalen M, Loreau M (2007b) Local facilitation, bistability and transitions in arid ecosystems. Theor Popul Biol 71:367-379. doi:10.1016/j.tpb.2006.09.003

Kéfi S, Rietkerk M, Roy M et al (2011) Robust scaling in ecosystems and the meltdown of patch size distributions before extinction. Ecol Lett 14:29-35. doi:10.1111/j.1461-0248.2010.01553.x

Kizaki S, Katori M (1999) Analysis of Canopy-Gap structures of forests by Ising-Gibbs states-equilibrium and scaling property of real forests. J Phys Soc Jpn 68:2553-2560. doi:10.1143/JPSJ.68.2553 
Kubo T, Iwasa Y, Furumoto N (1996) Forest spatial dynamics with gap expansion: total gap area and gap size distribution. J Theor Biol 180:229-246. doi:10.1006/jtbi.1996.0099

Lin Y, Han G, Zhao M, Chang SX (2010) Spatial vegetation patterns as early signs of desertification: a case study of a desert steppe in inner mongolia, China. Landsc Ecol 25:1519-1527. doi:10.1007/s10980-010-9520-z

Lucas PW, Turner IM, Dominy NJ, Yamashita N (2000) Mechanical defences to herbivory. Ann Bot 86:913-920. doi:10.1006/anbo.2000.1261

Maestre FT, Callaway RM, Valladares F, Lortie CJ (2009) Refining the stress-gradient hypothesis for competition and facilitation in plant communities. J Ecol 97:199-205. doi:10.1111/j.1365-2745.2008.01476.x

Manor A, Shnerb NM (2008) Facilitation, competition, and vegetation patchiness: from scale free distribution to patterns. J Theor Biol 253:838-842. doi:10.1016/j.jtbi.2008.04.012

Milchunas DG, Noy-Meir I (2002) Grazing refuges, external avoidance of herbivory and plant diversity. Oikos 99:113-130. doi:10.1034/j.1600-0706.2002.990112.x

Millennium Ecosystem Assessment (2005) Ecosystems and human well-being: desertification synthesis. World Resources Institute, Washington DC, USA

Moreno-de las Heras M, Saco PM, Willgoose GR, Tongway DJ (2011) Assessing landscape structure and pattern fragmentation in semiarid ecosystems using patch-size distributions. Ecol Appl 21:2793-2805. doi:10.1890/10-2113.1

Pascual M, Guichard F (2005) Criticality and disturbance in spatial ecological systems. Trends Ecol Evol 20:88-95. doi:10.1016/j.tree.2004.11.012

Pascual M, Roy M, Guichard F, Flierl G (2002) Cluster size distributions: signatures of selforganization in spatial ecologies. Philos Trans R Soc Lond Ser B Biol Sci 357:657-666. doi:10.1098/rstb.2001.0983

Puigdefábregas J (2005) The role of vegetation patterns in structuring runoff and sediment fluxes in drylands. Earth Surf Process Landf 30:133-147. doi:10.1002/esp.1181

Rietkerk M, Boerlijst MC, van Langevelde F et al (2002) Selforganization of vegetation in arid ecosystems. Am Nat 160:524530. doi:10.1086/342078
Rietkerk M, Dekker SC, De Ruiter PC, van de Koppel J (2004) Self-organized patchiness and catastrophic shifts in ecosystems. Science 305:1926-1929. doi:10.1126/science.1101867

Roy M, Pascual M, Franc A (2003) Broad scaling region in a spatial ecological system. Complexity 8:19-27. doi:10.1002/cplx.10096

Sala OE (1988) The effect of herbivory on vegetation structure. Plant form and vegetation structure, pp 317-330

Scanlon TM, Caylor KK, Levin SA, Rodriguez-Iturbe I (2007) Positive feedbacks promote power-law clustering of kalahari vegetation. Nature 449:209-212. doi:10.1038/nature06060

Scheffer M, Bascompte J, Brock WA et al (2009) Earlywarning signals for critical transitions. Nature 461:53-59. doi:10.1038/nature08227

Scheffer M, Carpenter S, Foley JA et al (2001) Catastrophic shifts in ecosystems. Nature 413:591-596. doi:10.1038/35098000

Suding KN, Gross KL, Houseman GR (2004) Alternative states and positive feedbacks in restoration ecology. Trends Ecol Evol 19:4653. doi:10.1016/j.tree.2003.10.005

Suding KN, Hobbs RJ (2009) Threshold models in restoration and conservation: a developing framework. Trends Ecol Evol 24:271-279. doi:10.1016/j.tree.2008.11.012

van de Koppel J, Rietkerk M, van Langevelde F et al (2002) Spatial heterogeneity and irreversible vegetation change in semiarid grazing systems. Am Nat 159:209-218. doi:10.1086/an.2002.159.issue-2

van de Koppel J, Rietkerk M, Weissing FJ (1997) Catastrophic vegetation shifts and soil degradation in terrestrial grazing systems. Trends Ecol Evol 12:352-356. doi:10.1016/S0169-5347(97)01133-6

von Hardenberg J, Kletter AY, Yizhaq H et al (2010) Periodic versus scale-free patterns in dryland vegetation. Proc R Soc B Biol Sci 277:1771-1776. doi:10.1098/rspb.2009.2208

WallisDeVries MF, Laca EA, Demment MW (1999) The importance of scale of patchiness for selectivity in grazing herbivores. Oecologia 121:355-363. doi:10.1007/s004420050939

Westoby M, Walker B, Noy-Meir I (1989) Opportunistic management for rangelands not at equilibrium. J Range Manag 266-274. doi: $10.2307 / 3899492$

White EP, Enquist BJ, Green JL (2008) On estimating the exponent of power-law frequency distributions. Ecology 89:905-912. doi:10.1890/07-1288.1 